\title{
Loss of spineless function transforms the Tribolium antenna into a thoracic leg with pretarsal, tibiotarsal, and femoral identity
}

\author{
Jane Patricia Toegel • Ernst A. Wimmer • \\ Nikola-Michael Prpic
}

Received: 20 June 2008 / Accepted: 29 October 2008 / Published online: 22 November 2008

(C) The Author(s) 2008. This article is published with open access at Springerlink.com

\begin{abstract}
The Drosophila spineless (ss) gene is regulated downstream of the appendage gene Distal-less $(D l l)$ and is involved in leg and antenna development. Specifically, loss of ss leads to the homeotic transformation of the arista, the distalmost antennal segment, into tarsal identity, and the loss or fusion of distal leg segments. Here we show that the ss homolog from the red flour beetle Tribolium castaneum also homeotically transforms the beetle antenna into leg, but the extent of the transformation is significantly larger than in Drosophila, as the entire antenna (except for the basal antennifer) is transformed into pretarsal, tibiotarsal, and femoral identity; i.e., the transformation comprises the $\mathrm{Dll}$ positive area in both appendages. We interpret the antennal phenotype in Tribolium as evidence for a more exclusive role of $s s$ in antennal determination downstream of $\mathrm{Dll}$ in the beetle. By contrast, the fact that, in Drosophila ss mutants, only a small portion of the Dll positive area in the antenna is homeotically transformed indicates that $\mathrm{Dll}$ uses additional targets to govern the development of the other antennal segments in the fly.
\end{abstract}

Communicated by S. Roth

J. P. Toegel $\cdot$ E. A. Wimmer $\cdot$ N.-M. Prpic $(\bowtie)$

Johann-Friedrich-Blumenbach Institute for Zoology

and Anthropology, Department of Developmental Biology,

Georg-August-University Goettingen,

GZMB, Ernst Caspari Building, Justus-von-Liebig-Weg 11,

37077 Goettingen, Germany

e-mail: nprpic@uni-goettingen.de

J. P. Toegel

e-mail: jtoegel@uni-goettingen.de

E. A. Wimmer

e-mail: ewimmer@gwdg.de
Keywords Morphological diversity .

Appendage development $\cdot$ Insect evolution .

Homeotic transformation $\cdot$ Selector genes

\section{Introduction}

Arthropod appendages are arguably the most diverse organs in the animal kingdom. Their form has been adapted to a large number of functions including locomotion, feeding, and sensing. The morphology of the appendages, however, does not only differ between species but also in one individual different appendage forms of different functions are present. In insects for example, the head bears three pairs of feeding appendages (mandibles, maxillae, labium) and one pair of sensory antennae, while the thorax bears three pairs of locomotory legs. All these appendages are believed to be serially homologous, i.e., they derive from a common ground state appendage [for an overview see Prpic and Damen (2008)].

Indeed the different appendage forms seem to share a common basic developmental program as they can easily be transformed into one another. The most famous example is the Antennapedia mutant in the vinegar fly Drosophila melanogaster in which the antennae are transformed into legs. This is caused by the ectopic expression of the Hox gene Antennapedia (Antp) in the antennal segment (Schneuwly et al. 1987). But there also are a number of other mutations that transform the antenna into leg, one of them being the aristapedia mutant (Struhl 1982; Burgess and Duncan 1990). In this case, only the distal portion of the antenna, the arista, is transformed into leg (hence the name), and the leg tissue of the transformation comprises only tarsal identity, but not more 
proximal elements. This Drosophila mutant phenotype is caused by the mutation of a single gene, spineless ( $s s$ ) (Duncan et al. 1998).

The antenna is one of the most diverse appendage types in insects, ranging from short few-segmented outgrowths to long whip-like appendages with dozens of segments. It is therefore unclear whether antennal morphology is governed by similar mechanisms in all insect species. Here we study the function of the ss gene in the beetle Tribolium castaneum and compare the results with the data from Drosophila. The adult fly antenna is short and stubby and consists of only four segments, whereas the Tribolium adult antenna is longer and thinner and consists of 11 segments. We find that the general effect of loss of $s s$ is the same in the two species, namely the transformation of antennal tissue into thoracic leg tissue. However, the amount to which antennal tissue is transformed differs significantly in the two species, pointing to differences in the details of $s s$ regulation and function. These evolutionary changes in the fine tuning of gene regulation might contribute to the morphological diversity of antennal morphology in insects.

\section{Materials and methods}

\section{Isolation of Tribolium spineless}

We searched the annotated genome sequence of Tribolium castaneum (Tribolium Genome Sequencing Consortium 2008) for an open reading frame with similarity to $s s$ from Drosophila, leading to the computer-annotated gene prediction GLEAN_11105. We then amplified by RTPCR (template cDNA has been prepared from embryos aged from 0 to $48 \mathrm{~h}$ ) a fragment of this predicted gene using the primers ss fw (AAG AGC AAC CCT AGC AAA CGT CAC CG) and $s s$ rev (TTC CTC TCT GAT CCA TCG AAA CCA AGG). The PCR fragments were cloned and three independent clones were sequenced all yielding identical sequences. The correspondence of the sequence of the clones with the GLEAN prediction was confirmed by pairwise alignments and the orthology of the fragments with Drosophila ss was established by phylogenetic analysis (data not shown).

Parental RNAi and whole-mount in situ hybridization

Probes for whole-mount in situ hybridization and double stranded RNA (dsRNA) were synthesized based on the fragment cloned with the primers given above. Both techniques were performed according to published protocols (Bucher et al. 2002; Prpic et al. 2001). The concentration of dsRNA that was injected into the pupae was $5,400 \mathrm{ng} / \mu \mathrm{l}$.

\section{Results and discussion}

\section{Expression of spineless in Tribolium}

In Tribolium, expression of ss during germ band elongation is restricted to the head appendages. Very strong expression is detected in the antennae, whereas weak staining is seen in the palps of both maxillae and labium (Fig. 1a,d,e). There is no expression of $s s$ in the mandible or in the thoracic legs at these stages. In fully elongated embryos during germ band retraction, additional expression domains appear (Fig. 1b). There is a complex, dot-like pattern in the lateral tissue that likely correlates with the developing peripheral nervous system. An additional expression domain also appears in the thoracic legs at their base in the form of a ring at the future junction between the coxa and the trochanter. There is, however, no expression in the distal portion of the thoracic legs. In older, fully retracted embryos (Fig. 1c), the pattern in the lateral tissue has increased in complexity. The proximal rings in the thoracic legs remain in thoracic segment 2 and 3, but the ring has disappeared in thoracic segment 1 . The by far strongest expression in all stages is always in the antennae (Fig. 1).

Parental RNAi with spineless replaces antennal segments for leg segments

We next studied the function of $s s$ using parental RNAi (pRNAi). In order to confirm that ss was indeed down regulated after pRNAi, we subjected embryos collected from pRNAi-treated mothers to whole-mount in situ hybridization using the ss RNA probe. In all treated embryos, we were not able to detect a signal (data not shown), indicating that ss expression was down regulated below the level of detection by in situ hybridization.

The wildtype larva displays a compact head capsule with the head appendages grouping together at the anterior (Fig. 2a), a thorax with three pairs of legs, and an abdomen with no appendages except for the posterior urogomphi (not shown). All appendages have a characteristic morphology and bear a number of stereotypic, diagnostic spines, bristles, and hairs that allow for unambiguous identification of appendage parts (Fig. 3a-c). The wildtype antenna (Fig. 3a) consists of the proximal antennifer, the scapus, the pedicellus, and the distal flagellum. The wildtype leg (cf. Figs. 2g and 3d) consists of the leg segments (podomeres) typical for insects, except that the tibia and tarsus are not yet separated forming the single podomere tibiotarsus.

In $100 \%$ of all analyzed larvae after pRNAi $(n=128)$, the antennae show a morphology that is very similar to a wildtype leg (Fig. 2b,c). The antennifer is normal, but the scapus, pedicellus, and the flagellum are replaced by the 
Fig. 1 Expression of $s s$ in Tribolium embryos. a Fully elongated germ band. b Embryo during mid-germ band retraction. c Fully retracted germ band. d Magnification of the head region of the embryo in (a). e Magnification of the head of an embryo at the start of retraction. Please note that the staining of the pleuropodium in $(\mathbf{b}, \mathbf{c})$ is an artifact seen with any probe at these developmental stages. All embryos are orientated with anterior to the left. Abbreviations: an antenna, $m d$ mandible, $m x$ maxilla, $l b$ labium, $t 1-t 3$ thoracic leg 1 to $3, p l$ pleuropodium

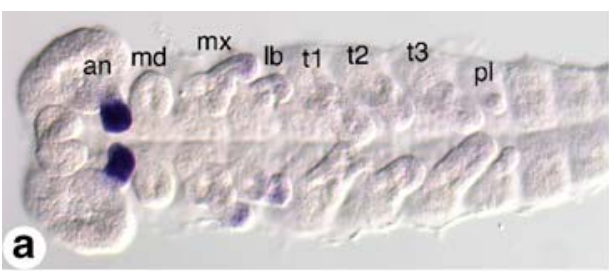

a
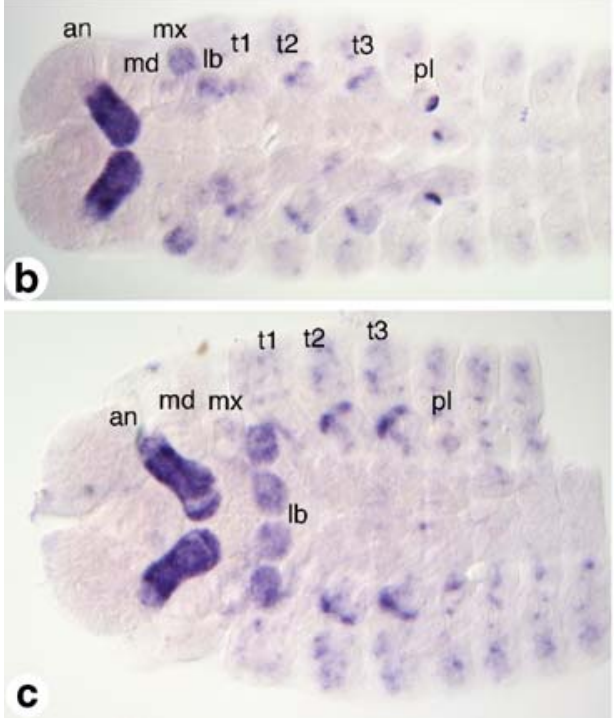
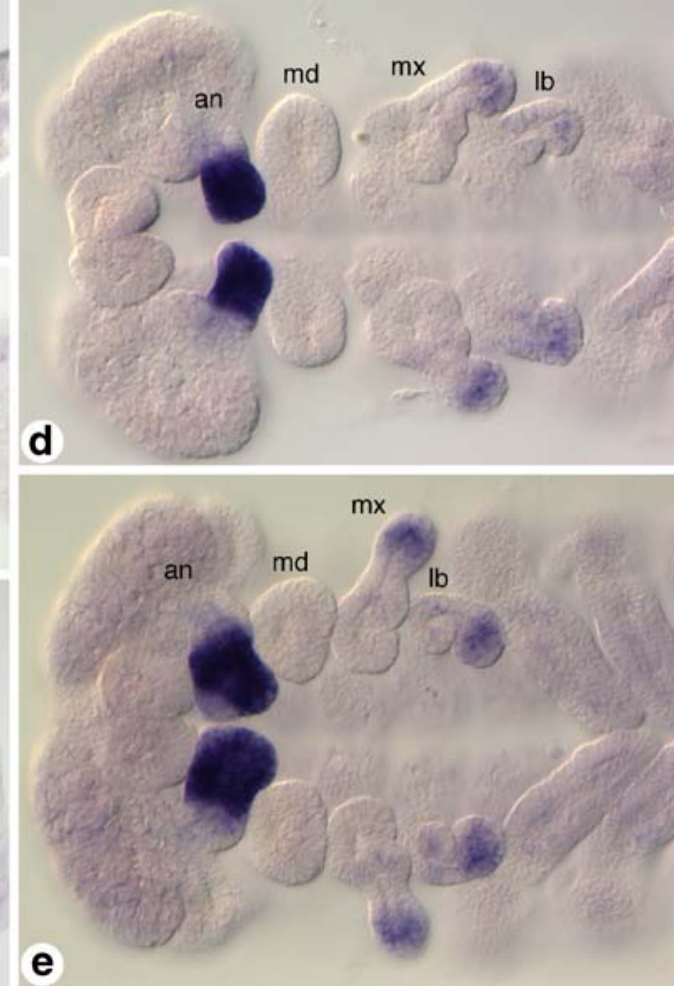
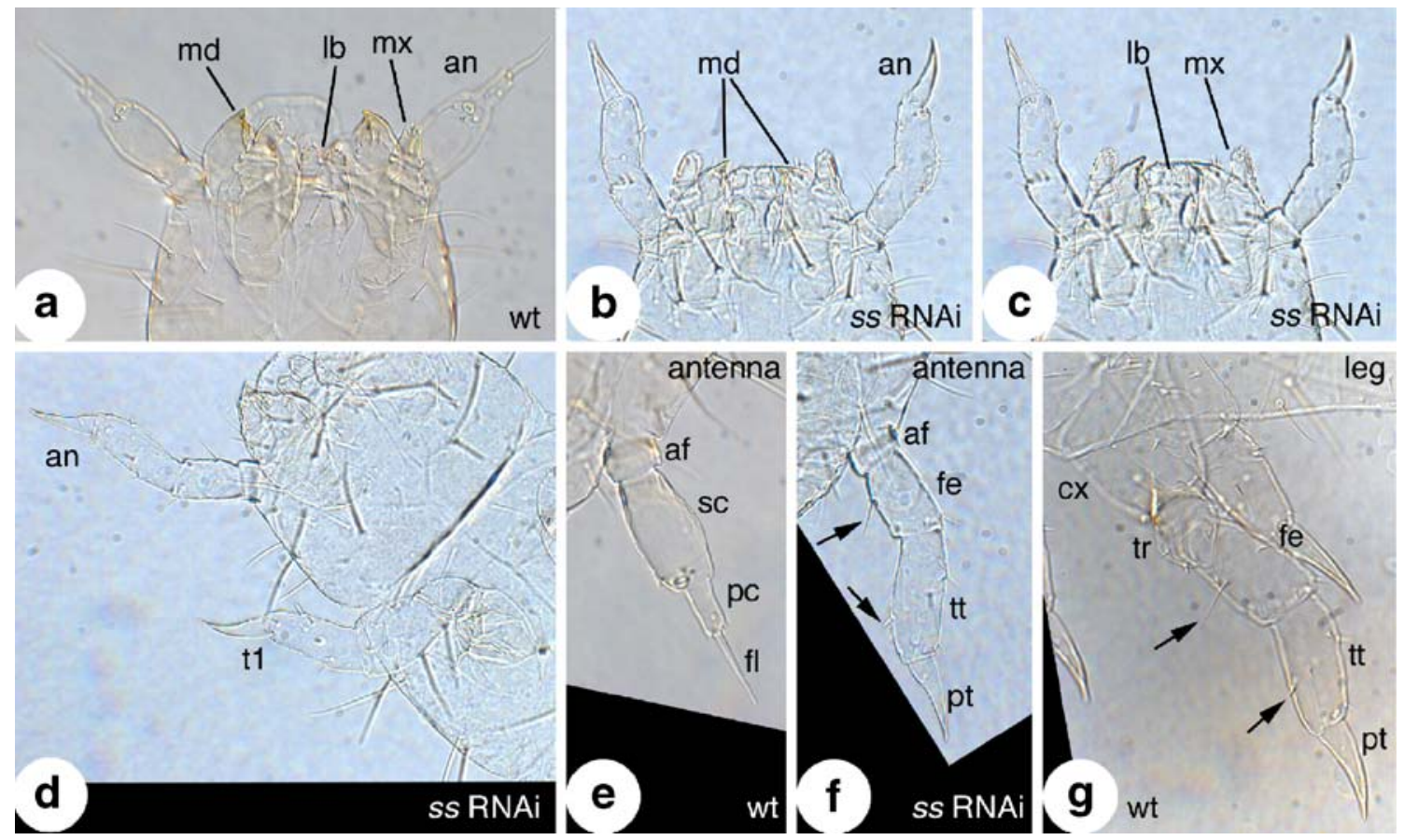

Fig. 2 Phenotypic effect of pRNAi with ss. a Head of a wildtype larva; the cephalic appendages are indicated. b Head of an ss RNAi phenocopy. The focus is on the antennae that are transformed into leg tissue. c Same as in (b), but focused on the gnathal appendages. Labium, maxillae, and mandibles are wildtype. (d) Anterior portion of an ss RNAi phenocopy. Note the similarity between the thoracic leg $(t 1)$ and the transformed antenna (an). The thoracic leg does not show abnormalities. e-g Comparison between wildtype antenna (e), transformed antenna of an ss RNAi phenocopy (f), and wildtype thoracic leg (g). Segments (podomeres) are indicated and the diagnostic bristles of femur and tibiotarsus are denoted with arrows. Abbreviations see Fig. 1. Additional abbreviations: af antennifer, $s c$ scapus, $p c$ pedicellus, $f l$ flagellum, $c x$ coxa, $t r$ trochanter, $f e$ femur, $t t$ tibiotarsus, pt pretarsus 


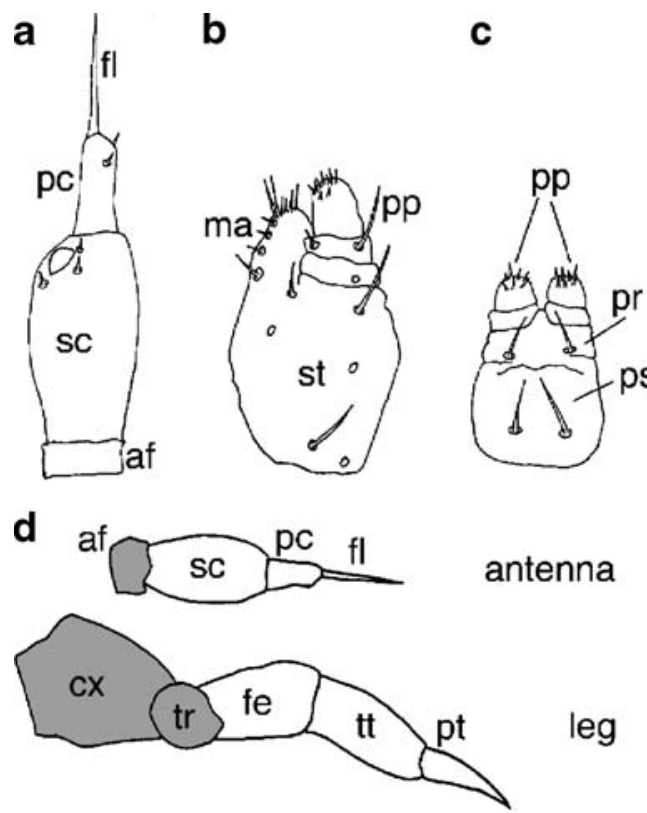

Fig. 3 Schematic drawings of Tribolium appendages, and areas that are transformed in ss RNAi phenocopies. a-c Morphology of larval head appendages of wildtype Tribolium, comprising characteristic segments and diagnostic bristles, hairs, and spines. The antenna (a) comprises the antennifer ( $a f)$, scapus $(s c)$, pedicellus $(p c)$, and flagellum $(f l)$. The maxilla (b) comprises the stipes $(s t)$, mala $(m a)$, and the palp ( $p p)$. The labium (c) comprises the postmentum ( $p s)$, prementum $(p r)$, and two palps $(p p)$. The morphology and bristle pattern of maxillae and labium is unchanged in ss RNAi phenocopies. d Parts of the antenna and leg that are not replaced or produced, respectively, by transformation are shaded in gray. The parts affected by homeotic transformation (white) largely correspond to the area also affected in $\mathrm{Dll}$ mutants. Additional abbreviations see Fig. 2

ectopic leg segments femur, tibiotarsus, and pretarsus (Fig. $2 \mathrm{e}-\mathrm{g}$ ). These ectopic podomeres are very similar to the normal leg podomeres. The ectopic pretarsus on the antenna is identical to the normal pretarsus which is characterized by its claw-like shape. The normal tibiotarsus is characterized by a ventral spine (arrow in Fig. $2 \mathrm{~g}$ ). The ectopic tibiotarsus also has this spine (Fig. 2f) and also bears all other stereotypic bristles making it indistinguishable from the normal tibiotarsus. The normal femur has a characteristic long ventral hair (arrow in Fig. $2 \mathrm{~g}$ ) and this hair is also present in the ectopic femur. The ectopic femur is virtually identical to the normal femur, except that it is somewhat shorter and thinner.

Although $s s$ is also expressed (at least in some stages) in maxilla, labium, and thoracic legs, we did not find any obvious phenotype in these appendages. The wildtype maxilla consists of a short palp, a tooth (mala), and a base (stipes) all with characteristic spines and bristles (Fig. 3b). The wildtype labium is a lip-like structure formed by fusion of the originally separate labial appendages. It consists of two proximal parts, postmentum and prementum, and two distal palps (Fig. 3c). Also, the labium has a number of characteristic bristles and spines. All these features were unchanged in the ss RNAi animals (Fig. 2b,c). All gnathal appendages (mandibles, maxillae, and labium) in the ss RNAi animals were of wildtype appearance. The thoracic legs of $s s$ RNAi animals also were indistinguishable from wildtype legs (Fig. 2d), including the area between the coxa and trochanter, where $s s$ is expressed during late embryonic stages.

Loss of Tribolium ss causes homeotic transformations of corresponding tissue

In ss null mutants in Drosophila a large portion of the antenna is affected. The third antennal segment (AIII) is malformed and lacks the bristles of the normal AIII segment and the arista is homeotically transformed into a
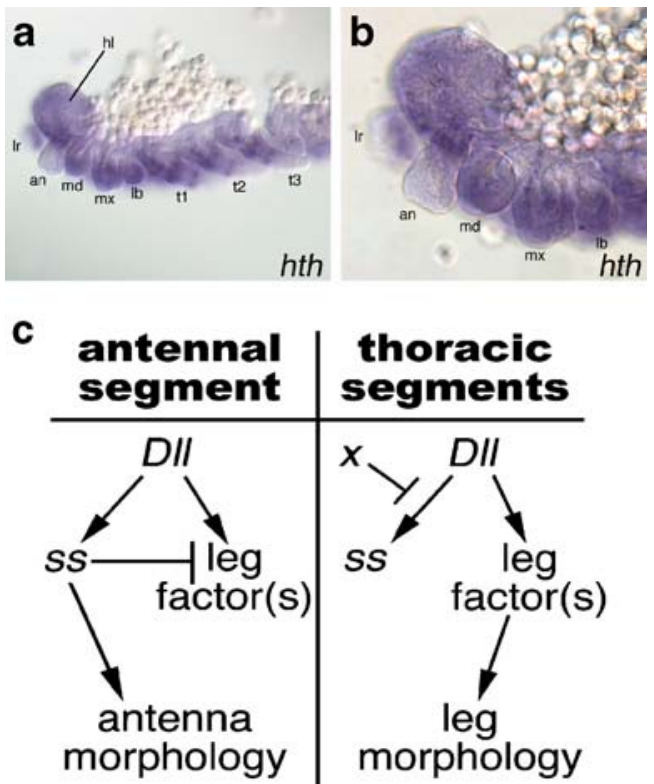

Fig. 4 Hypothetical regulation of $s s$ in Tribolium. a, b Expression of hth in Tribolium. a Head and thorax of an embryo during germ band retraction in lateral view. b Magnification of the head of the embryo in (a). In Drosophila, hth is expressed in most Dll expressing cells of the antenna and is regarded as the main antennal determinant. In contrast, Tribolium hth is restricted to the basis of the antenna which is similar to hth expression in the thoracic legs. It is unlikely, therefore, that Tribolium hth has a role as antennal determinant. c Hypothetical control of antennal versus leg development in Tribolium. Dll has a dual function in appendage development and is able to activate both ss (as the "antenna factor") and a hypothetical leg factor (or factors). In the antennal segment (left), ss represses the function of the leg factor leading to normal antenna development. In the thoracic segments, the activation of $s s$ by $D l l$ is inhibited by a hypothetical factor " $x$ ", leading to the exclusive activation of the leg factor and ensuing normal leg development. In this hypothetical framework, loss of ss would lead to the transformation of the $D l l$ expressing area of the antenna into leg, and would have no effect in the thoracic segments. These are the effects actually observed in Tribolium ss RNAi phenocopies. Abbreviations: $h l$ head lobe, $l r$ labrum. Additional abbreviations see Fig. 1 
tarsal segment and a claw (Duncan et al. 1998; Struhl 1982). Since the AIII segment is thought to be serially homologous to the femur and tibia of the thoracic legs (Postlethwait and Schneiderman 1971), the area affected by the loss of ss is comparable in Drosophila and Tribolium. However, because in Tribolium the entire affected area is transformed, the portion of the affected area that is homeotically transformed is significantly larger in Tribolium than in Drosophila.

In Tribolium, the proximal elements of both leg and antenna are never transformed or produced: the antennifer is always present and unaffected, and there is never an ectopic coxa or trochanter. This strongly suggests that the replacement of antennal tissue after loss of ss function in Tribolium affects only corresponding tissue in both appendage types (Fig. 3d). Thus, similar to Drosophila, loss of $s s$ function leads to a homeotic transformation of distal antennal tissue into distal leg tissue and the proximal portion of the antenna retains normal antennal identity.

The antennal transformation affects $D l l$ sensitive appendage portions

As noted above, the portion of the antenna that is transformed in Tribolium after the loss of ss function is larger than in Drosophila. Intriguingly, the area that is affected by the homeotic transformation is similar in both leg and antenna to the area that is lost in mutants of the Distal-less $(D l l)$ gene (Beermann et al. 2001). This suggests that in Tribolium ss might be the single master switch that distinguishes between the antenna promoting and the leg promoting function of $\mathrm{Dll}$ in the $\mathrm{Dll}$ positive area.

Indeed, it has been shown that ss is activated by $D l l$ and another factor, homothorax ( $h t h$ ), in the Drosophila antenna (Duncan et al. 1998; Dong et al. 2002; Emmons et al. 2007). The hth gene is regarded as the central antennal determinant in Drosophila, because it is expressed in almost all antennal cells, but is absent from the $D l l$ positive area in the thoracic legs, and loss of hth transforms antenna to leg (Casares and Mann 1998). The Tribolium hth gene (Prpic et al. 2003) is expressed in the entire mandible, maxilla, and labium, but is excluded from the $D l l$ area in antenna and leg (Fig. 4a, b). It is therefore unlikely that $h t h$ is the central antennal determinant in Tribolium. We hypothesize that in Tribolium Dll activates both ss and a (so far unidentified) leg factor (or factors) (Fig. 4c). In the antennal segment, ss represses the activation or function of the leg factor and thus leads to the formation of antennal morphology (left half of Fig. 4c). If $s s$ function is lost in this segment, the function of the leg factor is no longer repressed and the $D l l$ positive areas of the antenna are transformed into the corresponding leg parts, but more proximal regions of both appendages which are independent of Dll function can neither be replaced nor be produced. In the thoracic segments, an unknown factor (or factors) (" $\mathrm{x}$ " in Fig. 4c) completely represses the activating influence of $\mathrm{Dll}$ on $s s$ and $\mathrm{Dll}$ can only activate the leg factor leading to leg morphology (right half of Fig. 4c). Loss of $s s$ has thus no effect on the appendage phenotype in the larval thoracic segments. This hypothetical antenna-toleg transformation network in Tribolium would be somewhat different from the situation known from Drosophila. In Drosophila, ss is downstream of Dll, but loss of ss transforms only a subset of the $\mathrm{Dll}$ dependent tissue (Struhl 1982; Duncan et al. 1998; Emmons et al. 2007), indicating that $\mathrm{Dll}$ is using more than this one target gene to specify different antennal segments (e.g., Dong et al. 2002). Furthermore, there cannot be a complete repression of $s s$ activation in the thoracic segments in Drosophila, since ss is expressed in the $\mathrm{Dll}$ area of the thoracic legs and loss of ss function has a phenotype in the legs, leading to the loss of distal leg portions and the fusion of distal leg joints (Struhl 1982; Duncan et al. 1998).

\section{Conclusions}

Taken together, the data from Tribolium show that the overall function of ss in Tribolium and Drosophila is similar as both lead to homeotic transformations of the antennae to legs. However, the differences in the extent of the homeotic transformation point to differences in the regulation of $s s$ as well as its interplay with other factors. In particular, the relationship between Dll and ss might be different in Tribolium and Drosophila as discussed above. This notion is also supported by the fact that downregulation (but not loss) of Dll in Drosophila leads to homeotic transformations of the antennae that are very similar to the transformations seen in Drosophila ss mutants (e.g., Panganiban and Rubenstein 2002), whereas such transformations have never been observed in Tribolium Dll mutants or RNAi phenocopies (Beermann et al. 2001; Bucher et al. 2002).

Acknowledgements We thank Gregor Bucher, Johannes Schinko, and Nico Posnien for their support and help with the Tribolium work. The work has been supported by the European Community's Marie Curie Research Training Network ZOONET under contract MRTNCT-2004-005624 (EAW) and the Deutsche Forschungsgemeinschaft (NMP, grant number PR 1109/1-1).

Open Access This article is distributed under the terms of the Creative Commons Attribution Noncommercial License which permits any noncommercial use, distribution, and reproduction in any medium, provided the original author(s) and source are credited. 


\section{References}

Beermann A, Jay DG, Beeman RW, Hülskamp M, Tautz D, Jürgens G (2001) The Short antennae gene of Tribolium is required for limb development and encodes the orthologue of the Drosophila Distal-less protein. Development 128:287-297

Bucher G, Scholten J, Klingler M (2002) Parental RNAi in Tribolium (Coleoptera). Curr Biol 12:R85-R86

Burgess EA, Duncan I (1990) Direct control of antennal identity by the spineless-aristapedia gene of Drosophila. Mol Gen Genet 221:347-357

Casares F, Mann RS (1998) Control of antennal versus leg development in Drosophila. Nature 392:723-726

Dong PDS, Scholz Dicks J, Panganiban G (2002) Distal-less and homothorax regulate multiple targets to pattern the Drosophila antenna. Development 129:1967-1974

Duncan DM, Burgess EA, Duncan I (1998) Control of distal antennal identity and tarsal development in Drosophila by spinelessaristapedia, a homolog of the mammalian dioxin receptor. Genes Dev 12:1290-1303

Emmons RB, Duncan D, Duncan I (2007) Regulation of the Drosophila distal antenna determinant spineless. Dev Biol 302:412-426

Panganiban G, Rubenstein JLR (2002) Developmental functions of the Distal-less/Dlx homeobox genes. Development 129:4371-4386
Postlethwait JH, Schneiderman HA (1971) Pattern formation and determination in the antenna of the homeotic mutant Antennapedia of Drosophila melanogaster. Dev Biol 25:606-640

Prpic NM, Damen WGM (2008) Arthropod appendages: a prime example for the evolution of morphological diversity and innovation. In: Minelli A, Fusco G (eds) Evolving pathways: key themes in evolutionary developmental biology. Cambridge University Press, Cambridge, pp 381-398

Prpic NM, Wigand B, Damen WGM, Klingler M (2001) Expression of dachshund in wild-type and Distal-less mutant Tribolium corroborates serial homologies in insect appendages. Dev Genes Evol 211:467-477

Prpic NM, Janssen R, Wigand B, Klingler M, Damen WGM (2003) Gene expression in spider appendages reveals reversal of exd/hth spatial specificity, altered leg gap gene dynamics, and suggests divergent distal morphogen signaling. Dev Biol 264:119-140

Schneuwly S, Klemenz R, Gehring WJ (1987) Redesigning the body plan of Drosophila by ectopic expression of the homeotic gene Antennapedia. Nature 325:816-818

Struhl G (1982) Spineless-aristapedia: a homeotic gene that does not control the development of specific compartments in Drosophila. Genetics 102:737-749

Tribolium Genome Sequencing Consortium (2008) The first genome sequence of a beetle, Tribolium castaneum, a model for insect development and pest biology. Nature 452:949-955 